\title{
Editorial
}

\section{JAS Guideline and Statin Treatment}

It's very important to manage plasma lipids according to evidence derived from intervention studies, guidelines that have been established based on evidence, or a consensus among specialists in related fields of clinical science.

According to the Japan Atherosclerosis Society (JAS) Guidelines for Diagnosis and Treatment of Atherosclerotic Cardiovascular Diseases, the target levels of treatment for LDL-cholesterol are separately settled for six subject categories, which are defined by the number of accompanying risk factors for primary prevention and independently for secondary prevention.

Statin is a very useful cholesterol-lowering drug that is widely used around the world. The cholesterol-lowering activity of statins is variable depending on the chemical structures. The effect of statins on the prevention of coronary artery diseases (CAD) has been shown to depend largely on the cholesterol-lowering activity (1). However, previous intervention studies have also shown that the effect of statin on the prevention of CAD is not necessarily caused by the cholesterol level being reached with statin treatment. For example, in the WOS study (2), a sub-analysis of the prevention of CAD events compared the pravastatin and placebo subgroups with the same levels of LDL-cholesterol after interventional treatment. Frequency of events was significantly higher in the placebo group than in the pravastatin group. These findings suggest that the effect of statin on the prevention of CAD were derived from various conditions, such as differences in the subjects background and the pleiotropic effect of statins. Reaching a reduced LDL-cholesterol level by drug treatment may be, therefore, one of the markers for prevention of CAD as described by Nagashima $\mathrm{H}$, et al. in this issue (3). However, the target levels of LDL-cholesterol will not be ristricted to only JAS guideline, because the physicians will take care the patients with the various knowledges and strategies for treatment.

Again, it is very important for physicians to have guidelines for the treatment of patients. However, such guidelines should be considered one of the important opinions, and the final selection of the target level for LDLcholestero has to be made by the physicians themselves, considering severity and duration of risk factors, as well as other conditions of their patients.

\section{References}

(1) Cannon CP, Braunwald E, McCabe CH, Rader DJ, Rouleau JL, Belder R, Joyal SV, Hill KA, Pfeffer MA, and Skene AM: Pravastatin or Atorvastatin Evaluation and Infection Therapy-Thrombolysis in Myocardial Infarction 22 Investigators: Intensive versus Moderate Lipid Lowering with Statins after Acute Coronary Syndromes. N Engl J Med, 350: 14951504, 2004

( 2 ) The West of Scotland Coronary Prevention Study Group: Influence of pravastatin and plasma lipids on clinical events in the West of Scotland Coronary Prevention Study (WOSCOPS). Circulation, 97: 1440-1445, 1998

( 3 ) Nagashima $\mathrm{H}$ and Kasanuki $\mathrm{H}$ : The status of lipid management in 1836 patients with coronary artery disease. J Atheroscler Thromb, 12: 338-342, 2005

Yasushi Saito

Clinical Cell Biology

Graduate School of Med, Chiba University 